\title{
Metformin alters the insulin signaling pathway in ischemic cardiac tissue in a swine model of metabolic syndrome
}

\author{
Nassrene Y. Elmadhun, MD, Antonio D. Lassaletta, MD, Louis M. Chu, MD, and Frank W. Sellke, MD
}

Objective: The purpose of this study is to evaluate the effect of metformin on insulin signaling in ischemic cardiac tissue in a swine model of metabolic syndrome.

\begin{abstract}
Methods: Ossabaw miniswine were fed either a regular diet (Ossabaw control [OC]) or a hypercaloric diet (Ossabaw high cholesterol [OHC], Ossabaw high cholesterol with metformin [OHCM]). After 9 weeks, all animals underwent placement of an ameroid constrictor to the left circumflex artery to induce chronic ischemia. OHC animals were continued on a hypercaloric diet alone; the OHCM group was supplemented with metformin in addition to the hypercaloric diet. Seven weeks after ameroid placement, myocardial perfusion was measured and ischemic cardiac tissue was harvested for protein expression and histologic analysis.
\end{abstract}

Results: The OHC and OHCM groups had significantly higher body mass indices and serum insulin levels compared with the OC group. There were no differences in myocardial perfusion in the chronically ischemic territories. In the $\mathrm{OHC}$ group, there was upregulation of both an activator of insulin signaling insulin receptor substrate 1, and an inhibitor of insulin signaling phosphorylated insulin receptor substrate 2. In the OHCM group, there was upregulation of activators of insulin signaling including phosphorylated adenosine monophosphateactivated protein kinase $\alpha$, protein kinase B, phosphorylated protein kinase B, mammalian target of rapamycin, phosphorylated mammalian target of rapamycin, and phosphoinostitide 3-kinase, and upregulation of inhibitors including phosphorylated insulin receptor substrate 1, phosphorylated insulin receptor substrate 2, and retinol binding protein 4. Histologic analysis demonstrated increased expression of glucose transporter 1 at the plasma membrane in the OHCM group, but there was no difference in cardiomyocyte glycogen stores among groups.

Conclusions: Metformin treatment in the context of metabolic syndrome and myocardial ischemia dramatically upregulates the insulin signaling pathway in chronically ischemic myocardium, which is at the crossroads of known metabolic and survival benefits of metformin. (J Thorac Cardiovasc Surg 2013;145:258-66)

Despite advances in treatment, diabetes mellitus still affects 25.8 million Americans and is a major cause of morbidity and mortality in the United States. ${ }^{1}$ Patients with diabetics often experience a group of metabolic derangements including dyslipidemia, hypertension, and obesity (also known as metabolic syndrome), which more than doubles their risk of developing cardiovascular disease. ${ }^{2-5} \mathrm{~A}$ central component in the development of type 2 diabetes mellitus and metabolic syndrome is insulin resistance. ${ }^{6}$ One of the mainstay treatments of type 2 diabetes mellitus

From the Division of Cardiothoracic Surgery, Cardiovascular Research Center, Warren Alpert Medical School of Brown University, Providence, RI.

Funding for this research was provided by the National Heart, Lung, and Blood Institute (R01HL46716, R01HL69024, and R01HL85647; to Dr Sellke), National Institutes of Health (NIH) training grant 5T32-HL076134 (to Dr Lassaletta), and NIH training grant 5T32-HL094300-03 (to Dr Chu and Dr Elmadhun).

Disclosures: Authors have nothing to disclose with regard to commercial support.

Read at the 92nd Annual Meeting of The American Association for Thoracic Surgery, San Francisco, California, April 28-May 2, 2012.

Received for publication April 6, 2012; revisions received July 24, 2012; accepted for publication Sept 13, 2012; available ahead of print Oct 22, 2012.

Address for reprints: Frank W. Sellke, MD, Division of Cardiothoracic Surgery, Cardiovascular Research Center, Warren Alpert Medical School of Brown University, 2 Dudley St, MOC 360, Providence, RI 02905 (E-mail: fsellke@ lifespan.org).

$0022-5223 / \$ 36.00$

Copyright $(2) 2013$ by The American Association for Thoracic Surgery

http://dx.doi.org/10.1016/j.jtcvs.2012.09.028 is metformin, an orally administered biguanide. Metformin reduces blood glucose levels by reducing hepatic glucose production and increasing peripheral glucose uptake. ${ }^{7}$ Metformin also has direct cardioprotective properties that are independent of its antihyperglycemic effects. Epidemiologic studies have shown that patients with type 2 diabetes mellitus treated with metformin had reduced all-cause mortality. ${ }^{8,9}$ In animal studies, metformin has been shown to reduce infarct size and attenuate myocardial remodeling, preserve myocardial function, limit cardiac hypertrophy, and reduce the development of heart failure after myocardial infarction. ${ }^{10}$

Studies have shown that the cardioprotective properties of metformin are mediated by protein kinase B (AKT) and adenosine monophosphate-activated protein kinase (AMPK) by promoting cell survival during periods of ischemia. ${ }^{10}$ Interestingly, both AKT and AMPK are also key protein kinases in the insulin signaling cascade. Therefore, it is prudent to investigate further the effects of metformin at the junction of its cardioprotective and glucose lowering effects: the insulin signaling cascade. We developed a clinically relevant swine model of metabolic syndrome and chronic myocardial ischemia to investigate the effects of metformin on the insulin signaling pathway in ischemic myocardium. 


$\begin{aligned} & \text { Abbreviations and Acronyms } \\ & \text { ACC }=\text { acetyl coenzyme A carboxylase } \\ & \text { AKT }=\text { protein kinase B } \\ & \text { AMP }=\text { adenosine monophosphate } \\ & \text { AMPK }=\text { adenosine monophosphate-activated } \\ & \text { protein kinase } \\ & \text { ATP }=\text { adenosine triphosphate } \\ & \text { CoA }=\text { coenzyme A } \\ & \text { FAS }=\text { fatty acid synthase } \\ & \text { FOX01 }=\text { foxhead box 01 } \\ & \text { GLUT1 }=\text { glucose transporter } 1 \\ & \text { GLUT4 }=\text { glucose transporter } 4 \\ & \text { IRS }=\text { insulin receptor substrate } \\ & \text { MTOR }=\text { mammalian target of rapamycin } \\ & \text { OC }=\text { Ossabaw control } \\ & \text { OHC } \text { Ossabaw high cholesterol } \\ & \text { OHCM }=\text { Ossabaw high cholesterol with } \\ & \text { metformin } \\ & \text { PI3K }=\text { phosphoinostitide } 3 \text {-kinase } \\ & \text { p-IRS }=\text { phosphorylated insulin receptor } \\ & \text { substrate } \\ & \text { p-MTOR } \text { phosphorylated mammalian target of } \\ & \text { rapamycin } \\ & \text { RBP4 }=\text { retinol binding protein } 4\end{aligned}$

\section{METHODS}

\section{Animal Model}

Twenty-four intact male Ossabaw miniswine (Purdue Ossabaw Facility, Indiana University, Indianapolis, Ind) were divided into 3 groups according to diet at 6 weeks of age. The control group was fed $500 \mathrm{~g} / \mathrm{day}$ of regular chow (Ossabaw control [OC], $\mathrm{n}=8$ ). The high-cholesterol animals (Ossabaw high cholesterol [OHC], $\mathrm{n}=8$ ) were fed $500 \mathrm{~g} /$ day of high-cholesterol chow consisting of $4 \%$ cholesterol, $17.2 \%$ coconut oil, $2.3 \%$ corn oil, $1.5 \%$ sodium cholate, and $75 \%$ regular chow (Sinclair Research, Columbia, Mo). High-cholesterol metformin animals (Ossabaw high cholesterol with metformin $[\mathrm{OHCM}], \mathrm{n}=8$ ) were also fed high-cholesterol chow. After 9 weeks of diet initiation, all animals underwent surgical placement of an ameroid constrictor to induce chronic myocardial ischemia (see Surgical Interventions). Postoperatively, the OHCM group was supplemented with $500 \mathrm{mg}$ metformin orally twice daily and all animals were continued on their respective diet. Seven weeks after ameroid constrictor placement, all animals were weighed and underwent functional cardiac and hemodynamic measurements, euthanasia, and cardiac tissue harvest. All animals were observed to ensure complete consumption of food and supplement, had unlimited access to water, and were housed in a warm, nonstressful environment for the duration of the experiment.

\section{Surgical Interventions}

Anesthesia. Anesthesia was induced with an intramuscular injection of telazol $(4.4 \mathrm{mg} / \mathrm{kg})$. Animals were intubated endotracheally and ventilated mechanically at 12 to 20 breaths per minute, and general anesthesia was maintained with a gas mixture of oxygen at 1.5 to $2 \mathrm{~L} /$ minute and isoflurane at $0.75 \%$ to $3.0 \%$ concentration.

Ameroid constrictor placement. Animals were given a single dose of intravenous enrofloxacin $5 \mathrm{mg} / \mathrm{kg}$ for antibiotic prophylaxis, and general anesthesia was induced and maintained. Animals were prepped and draped in the usual sterile fashion. The heart was exposed through a left minithoracotomy and pericardiotomy. The left atrial appendage was retracted and the left circumflex artery was dissected at the takeoff of the left main coronary artery. The circumflex artery was occluded for 2 minutes, during which time $5 \mathrm{~mL}$ isotope-labeled gold microspheres (BioPhysics Assay Laboratory, Worcester, Mass) was injected into the left atrium to establish shadow labeling of the ischemic myocardium. The ameroid constrictor was placed around the left circumflex artery (Research Instruments SW, Escondito, Calif). The pericardium was reapproximated loosely followed by a layered closure of the surgical incision. Postoperative pain was controlled with a single dose of intramuscular Buprenorphine $(0.03 \mathrm{mg} / \mathrm{kg})$ and a 72 -hour Fentanyl patch $(4 \mu \mathrm{g} / \mathrm{kg})$. All animals received $325 \mathrm{mg}$ aspirin daily starting 1 day preoperatively and continuing for a total of 5 days for prophylaxis against thromboembolic events. All animals continued perioperative antibiotics of enrofloxacin 68 mg orally daily for 5 days.

Cardiac harvest. Under general anesthesia, coronary angiography of the left and right coronary circulation was performed as described previously. ${ }^{11}$ Complete occlusion of the left circumflex artery was confirmed angiographically in all cases. A blinded interventional cardiologist then assessed the TIMI, Rentrop, and Blush scores. The heart was exposed via a median sternotomy. Global and regional myocardial function and contractility were measured by placing sonomicrometer crystals (Sonometrics Corporation, Ontario, Canada) in the normally perfused left ventricle as described previously. ${ }^{11}$ Pressure catheters were also placed in the descending aorta through a right femoral sheath and into the left ventricle through an apical puncture. ${ }^{11}$ After microsphere injection (see Myocardial Perfusion), animals were euthanized by exsanguination, and chronically ischemic myocardial samples in the left circumflex territory were collected for further analysis. The Institutional Animal Care and Use Committee of the Rhode Island Hospital approved all experiments. Animals were cared for in compliance with the Principles of Laboratory Animal Care formulated by the National Society for Medical Research and the Guide for the Care and Use of Laboratory Animals. ${ }^{12}$

\section{Serologic Studies}

Blood samples were drawn from the jugular vein prior to euthanasia and tissue harvest for insulin measurement. The chemistry laboratory at the Rhode Island Hospital, Providence, RI, analyzed the serum samples.

\section{Myocardial Perfusion}

Myocardial perfusion was measured by injecting gold isotope-labeled microspheres (Biophysics Assay Laboratory) into the left atrium at the time of ameroid placement during a brief left circumflex artery occlusion. At the final operation, prior to cardiac harvest, lutetium microspheres were injected while simultaneously withdrawing blood from a femoral artery catheter. Samples of the left ventricle and blood were dried at $60^{\circ} \mathrm{C}$ for $>48$ hours, and microsphere density was quantified with a gamma counter after exposure to neutron bean radiation (Biophysics Assay Laboratory). Myocardial blood flow to each sample was calculated using the following equation:

$$
\text { Blood flow }=\frac{\text { Withdrawal rate }}{\text { Tissue weight }} \times \frac{\text { Tissue microsphere count }}{\text { Blood microsphere count }}
$$

\section{Protein Expression}

Forty micrograms of the radio-immunoprecipitation assay (Boston BioProducts, Ashland, Mass) soluble fraction of myocardial lysates from the chronically ischemic territory were fractionated by SDS-PAGE using 3\% to $8 \%$ Tris-acetate gel (NuPage Novex Mini Gel; Invitrogen, Carlsbad, Calif) for molecular weight targets $>100 \mathrm{kDa}$ and $4 \%$ to $12 \%$ Bis-Tris 

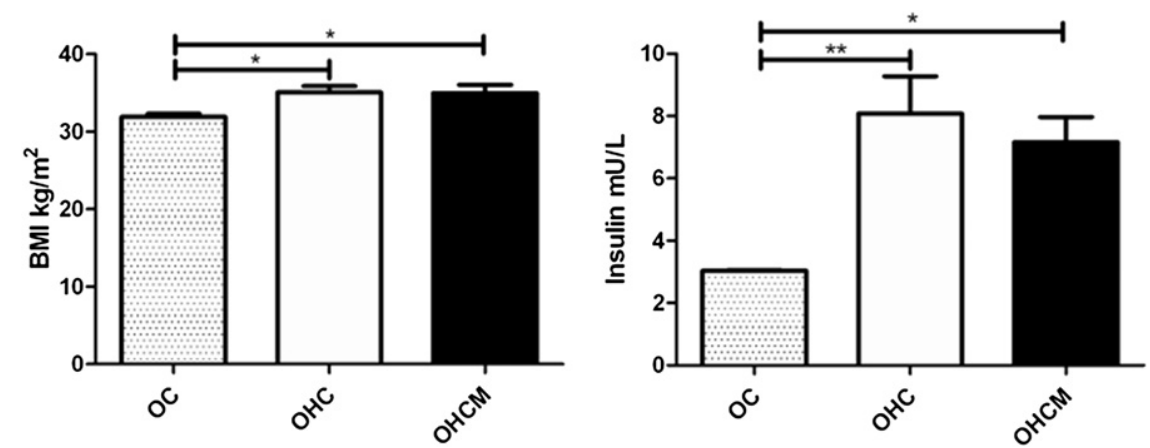

FIGURE 1. Comparative BMI and serum insulin levels in Ossabaw control $(O C)$, Ossabaw high cholesterol (OHC), Ossabaw high cholesterol with metformin $(O H C M)$. *Bonferroni $P<.05$. **Bonferroni $P<.01$. BMI, Body mass index.

gels for molecular weight targets $<100 \mathrm{kDa}$ (NuPage Novex Mini Gel). The protein was then transferred to polyvinylidene difluoride membranes (Millipore, Bedford, Mass) and incubated overnight at $4{ }^{\circ} \mathrm{C}$ with primary antibodies at dilutions recommended by the manufacturer against phosphorylated insulin receptor substrate 1 (p-IRS1) (Ser 612), IRS1, IRS2, phosphorylated AKT (Thr 473), AKT, phosphorylated AMPK $\alpha$ (Thr 172), AMPK $\alpha$, phosphorylated forkhead box 01 (FOX01) (Ser 256), FOX01, phosphorylated mammalian target of rapamycin (p-MTOR) (Ser 2481), MTOR, phosphoinostitide 3-kinase (PI3K), acetyl coenzyme A (CoA) carboxylase (ACC), phosphorylated ACC (Ser 79), S6, phosphorylated S6 (Ser 235/236) (all from Cell Signaling, Danvers, Mass); retinol binding protein 4 (RBP4; Abnova, Taipei, Taiwan); p-IRS2 (Ser 731) and glucose transporter 1 (GLUT1; from Abcam, Cambridge, Mass); Deptor (Sigma-Aldrich, St. Louis, Mo); and fatty acid synthase (FAS) (Epitomics, Burlingame, Calif). Membranes were incubated with the appropriate horseradish peroxidaselinked secondary antibody for 1 hour at room temperature (Jackson ImmunoResearch, West Grove, Pa). Immune complexes were visualized with enhanced chemiluminescence and images were captured with a digital camera system (G-Box; Syngene, Cambridge, UK). Band densitometry was quantified as arbitrary light units using Image-J software (National Institutes of Health, Bethesda, Md). All membranes were probed with glyceraldehyde 3-phosphate dehydrogenase (cell signaling) to correct for loading error.

\section{Immunohistochemical Analysis}

Frozen myocardium from the chronically ischemic territory was sectioned (thickness, $12 \mu \mathrm{m}$ ) and fixed in $10 \%$ formalin for 10 minutes. Sections were blocked with $1 \%$ bovine serum albumin in phosphatebuffered saline for 1 hour at room temperature and incubated with anti-GLUT 1 antibody (Abcam) overnight at $4^{\circ} \mathrm{C}$. Sections were then incubated with DyLight 549-conjugated antirabbit antibody (Jackson ImmunoResearch) for 45 minutes, then mounted with Vectashield with 4',6-diamidino-2-phenylindole (Vector Laboratories, Burlingame, Calif). Images were captured at $20 \times$ magnification with a Nikon E800 Eclipse microscope (Nikon, Tokyo, Japan) at the same exposure. Brightness was enhanced identically in all images to display immunofluorescence optimally (Adobe Photoshop, San Jose, Calif).

\section{Histology}

Periodic acid-Schiff staining was performed on frozen tissue sections of the chronically ischemic myocardium by the Pathology and Histology Core Facility at Rhode Island Hospital. Periodic acid-Schiff images were obtained using Aperio ScanScope technology (Vista, Calif) and captured at $20 \times$ magnification.

\section{Data Analysis}

All results are reported as mean \pm standard error of the mean. A 1-way analysis of variance was used to compare the means among groups, followed by a post hoc Bonferroni test to compare the means between groups using GraphPad Prism 5.0 software (GraphPad Software, Inc, San Diego, Calif).

\section{RESULTS}

\section{Animal Model}

All animals included in the analysis survived the entire experiment. One animal in the OC group and 1 in the OHC group died postoperatively, the first 8 days postoperatively and the second 2 days postoperatively. Necropsy did not reveal a clear cause of death, and it was assumed the animals died after an acute arrhythmia leading to sudden cardiac death. Another animal in the OHCM group was euthanized after a prolonged myocardial infarction at the time of ameroid placement. The animals that did not survive to completion of the experiment were excluded from analysis and replaced with new animals. At the time of the final operation, animals were weighed and the body mass index was calculated. Although all animals were obese, the OHC and OHCM groups had significantly higher body mass indexes compared with the OC group $\left(35.09 \mathrm{~kg} / \mathrm{m}^{2}, 34.97 \mathrm{~kg} / \mathrm{m}^{2}\right.$, and $31.91 \mathrm{~kg} / \mathrm{m}^{2}$, respectively). Serum insulin levels in the OC group were significantly lower compared with the $\mathrm{OHC}$ and OHCM groups $(3.04 \mathrm{mU} / \mathrm{L}, 8.08 \mathrm{mU} / \mathrm{L}$, and $7.16 \mathrm{mU} / \mathrm{L}$, respectively; Figure 1).

There was no difference in the TIMI score of coronary blood flow, Rentrop scores of the left and right coronary circulation, or Blush scores between any of the groups (data reported previously by Lassaletta and colleagues ${ }^{11}$ ). Interestingly, by gross observation, there was no or minimal subendocardial signs of infarction. Thus, this model is one of chronic ischemia and not infarction.

\section{Myocardial Perfusion}

Myocardial perfusion to the chronically ischemic territory was measured at the time of the final operation and there were no differences in flow in the $\mathrm{OC}, \mathrm{OHC}$, and $\mathrm{OHCM}$ groups $(0.50 \pm 0.04 \mathrm{~mL} / \mathrm{min} / \mathrm{g}, 0.58 \pm 0.08 \mathrm{~mL} / \mathrm{min} / \mathrm{g}$, and $0.67 \pm 0.15 \mathrm{~mL} / \mathrm{min} / \mathrm{g}$, respectively; analysis of variance, $P=.611$ ). Lassaletta and colleagues ${ }^{11}$ previously reported that in this animal model there was significant 
decrease in the developed left ventricular pressure in the $\mathrm{OC}$ versus the $\mathrm{OHC}$ group ( $56 \mathrm{~mm} \mathrm{Hg}$ vs $98 \mathrm{~mm} \mathrm{Hg}$ ), and a significant increase in the MAP in the OHC group versus the $\mathrm{OC}$ and $\mathrm{OHCM}$ groups $(112 \mathrm{~mm} \mathrm{Hg}$ vs $85 \mathrm{~mm} \mathrm{Hg}$ and 68 $\mathrm{mm} \mathrm{Hg}$, respectively). There was no difference in measured global or regional left ventricular contractility. ${ }^{11}$ Also, there was no difference in microvessel reactivity to endotheliumdependent vasodilator adenosine diphosphate, but there was a significant increase in microvessel reactivity to endothelium-independent vasodilator SNP in the OHC and OHCM groups. ${ }^{11}$ Despite these differences, there was no change in the measured myocardial perfusion in this study.

\section{Protein Expression}

Western blot analysis in the ischemic territories demonstrated upregulation in IRS1, FOX01, and PI3K in the OHC group compared with the OC group. There was a significant increase in expression of p-IRS1 (Ser 612), p-IRS2 (Ser 731), phosphorylated AMPK $\alpha$ (Thr 172), AKT, phosphorylated AKT (Ser 473), FOX01, phosphorylated FOX01 (Ser 256), MTOR, p-MTOR (Ser 2481), PI3K, RBP4, Deptor, and pS6 (Ser 235/236) in the OHCM group compared with the OC group. There was also a significant increase in p-IRS2 (Ser 731), phosphorylated AMPK $\alpha$ (Thr 172), phosphorylated AKT (Ser 473), phosphorylated S6 (Ser 235/236), MTOR, and p-MTOR (Ser 2481) expression in the OHCM group compared with the OHC group (Table 1 and Figure 2).

\section{Histologic Analysis}

Immunohistochemical staining for GLUT1 demonstrated significantly increased membrane-bound GLUT1 expression in the $\mathrm{OHC}$ and $\mathrm{OHCM}$ groups compared with the OC group $(2.19,3.76$, and 1.00 integrated optical density expressed as fold change with respect to OC, respectively). Periodic acid-Schiff staining was homogenous and similar in all 3 groups (Figure 3).

\section{DISCUSSION}

In previous animal studies, we have established that Ossabaw miniswine fed a hypercaloric/hypercholesterolemic diet develop metabolic syndrome, including dyslipidemia, hypertension, and glucose intolerance. ${ }^{11}$ In this study, all animals were obese, and the OHC and OHCM groups had significantly higher body mass indices compared with the OC group, which is expected because the hypercaloric diet provides $24 \%$ more calories than regular chow. The $\mathrm{OHC}$ and OHCM groups also had significantly higher serum insulin levels, which is consistent with our previous studies in which animals fed a hypercaloric diet develop hyperinsulinemia. The addition of metformin did not alter the measured insulin levels significantly.

This study demonstrates that metformin supplementation in a swine model of metabolic syndrome alters significantly
TABLE 1. Western blot analysis of $\mathrm{OHC}$ and $\mathrm{OHCM}$ protein expression in ischemic myocardium

\begin{tabular}{|c|c|c|c|c|}
\hline & OHC & OHCM & $\begin{array}{l}\text { ANOVA } \\
P \text { value }\end{array}$ & $\begin{array}{c}\text { Bonferroni } \\
P \text { value }\end{array}$ \\
\hline IRS1 & $1.39 \pm 0.10$ & $1.29 \pm 0.15$ & .048 & $<.05^{*}$ \\
\hline p-IRS1 (Ser 612) & $1.43 \pm 0.10$ & $1.74 \pm 0.31$ & .003 & $<.01 \dagger$ \\
\hline IRS2 & $1.11 \pm 0.07$ & $1.25 \pm 0.08$ & .289 & $>.05$ \\
\hline p-IRS2 (Ser 731) & $1.08 \pm 0.50$ & $1.43 \pm 0.05$ & .002 & $<.01 \dagger,<.05 \ddagger$ \\
\hline $\mathrm{AMPK} \alpha$ & $0.93 \pm 0.11$ & $0.95 \pm 0.11$ & .944 & $>.05$ \\
\hline $\begin{array}{l}\text { p-AMPK } \alpha \\
\text { (Thr 172) }\end{array}$ & $0.98 \pm 0.078$ & $30.68 \pm 2.09$ & $<.0001$ & $<.001 \dagger \ddagger$ \\
\hline $\mathrm{AKT}$ & $1.54 \pm 0.38$ & $2.26 \pm 0.35$ & .030 & $<.05 \dagger$ \\
\hline p-AKT (Ser 473) & $1.04 \pm 0.15$ & $14.02 \pm 1.46$ & $<.0001$ & $<.001 \dagger \ddagger$ \\
\hline FOX01 & $1.48 \pm 0.15$ & $1.59 \pm 0.13$ & .004 & $<.05^{*},<.01 \dagger$ \\
\hline p-FOX01 (Ser 256) & $2.45 \pm 0.61$ & $3.19 \pm 0.60$ & .020 & $<.05 \dagger$ \\
\hline MTOR & $1.40 \pm 0.27$ & $2.54 \pm 0.19$ & .0003 & $<.001 \dagger,<.01 \dagger$ \\
\hline $\begin{array}{l}\text { p-MTOR } \\
\quad(\text { Ser 2481) }\end{array}$ & $1.59 \pm 0.36$ & $6.96 \pm 1.03$ & $<.0001$ & $<.001 \dagger \ddagger$ \\
\hline PI3K & $1.44 \pm 0.07$ & $1.34 \pm 0.17$ & .003 & $<.01^{*},<.05 \dagger$ \\
\hline RBP4 & $1.67 \pm 0.17$ & $2.70 \pm 0.58$ & .004 & $<.01 \dagger$ \\
\hline GLUT4 & $0.85 \pm 0.12$ & $1.06 \pm 0.22$ & .629 & $>.05$ \\
\hline $\mathrm{ACC}$ & $0.77 \pm 0.08$ & $0.94 \pm 0.17$ & .37 & $>.05$ \\
\hline p-ACC (Ser 79) & $0.97 \pm 0.05$ & $1.02 \pm 0.10$ & .69 & $>.05$ \\
\hline FAS & $0.82 \pm 0.16$ & $1.03 \pm 0.13$ & .42 & $>.05$ \\
\hline S6 & $1.08 \pm 0.09$ & $0.95 \pm 0.06$ & .38 & $>.05$ \\
\hline p-S6 (Ser 235/236) & $0.98 \pm 0.07$ & $0.59 \pm 0.07$ & .01 & $<.05 \dagger,<.05 \ddagger$ \\
\hline Deptor & $1.09 \pm 0.03$ & $1.21 \pm 0.05$ & .01 & $<.01 \dagger$ \\
\hline \multicolumn{5}{|c|}{ 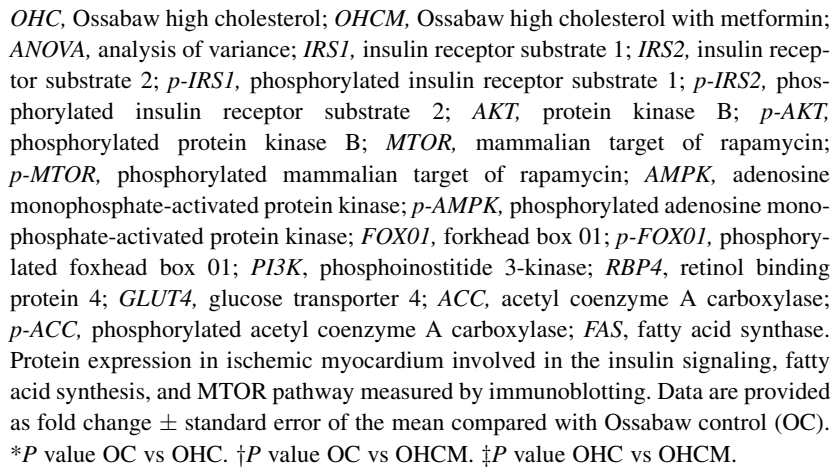 } \\
\hline
\end{tabular}

the insulin signaling pathway in ischemic myocardium. The insulin signaling cascade is initiated when insulin binds the insulin receptor, resulting in tyrosine phosphorylation of IRS, which activates IRS and allows it to bind to PI3K. In turn, PI3K activates AKT, which triggers the translocation of intracellular glucose transporter 4 (GLUT4) vesicles to the plasma membrane. Once docked at the plasma membrane, GLUT4 takes up glucose, improves glucose utilization, and reduces serum glucose levels. ${ }^{13}$ In a well-fed state, AMPK and MTOR are activated, which further propagate the insulin signaling pathway (Figure 4).

Insulin receptor substrate 1 and IRS2 are widely expressed, with IRS1 as the predominant isoform in muscle and IRS2 found mainly in the liver, muscle, and adipose tissue. ${ }^{14}$ In this study, there was upregulation of IRS1 in the $\mathrm{OHC}$ group and upregulation of the p-IRS2 (Ser 731) in 

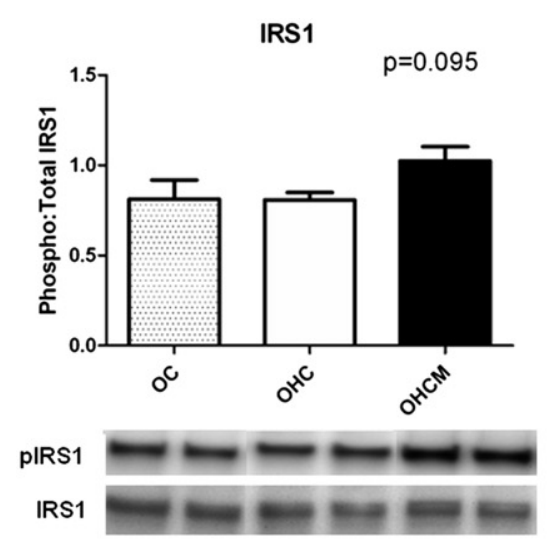

MTOR
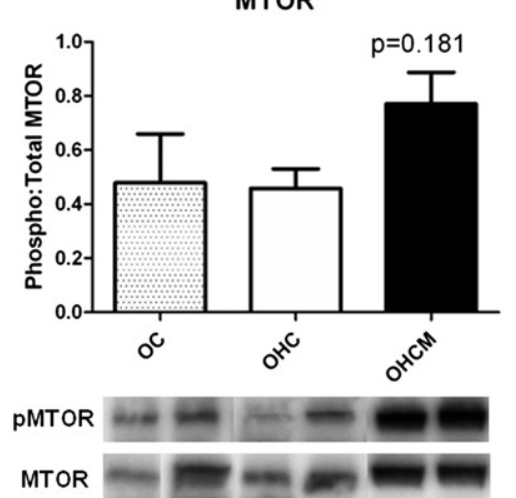

IRS2
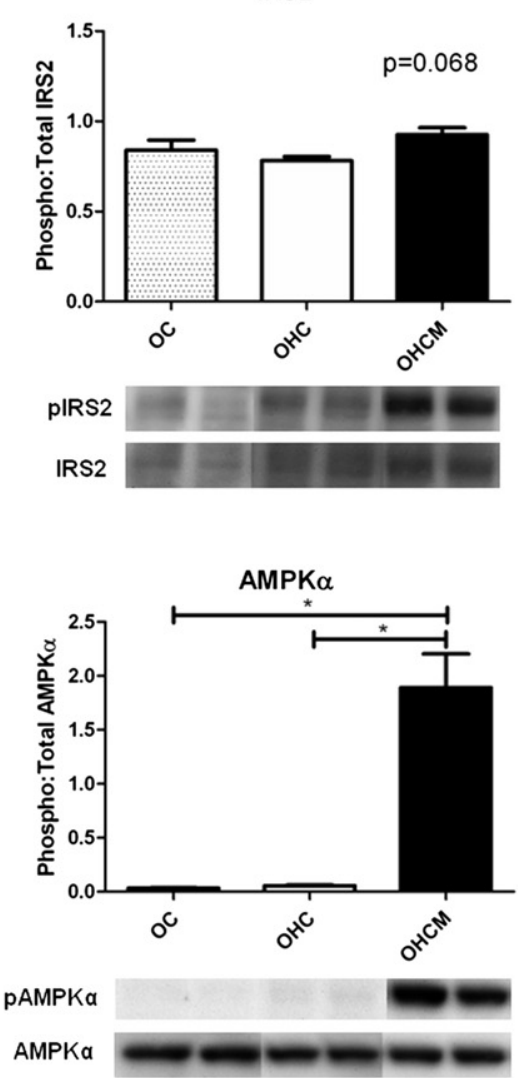

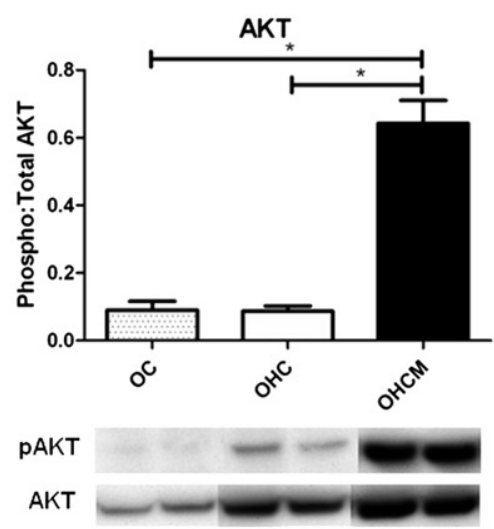

Fox01

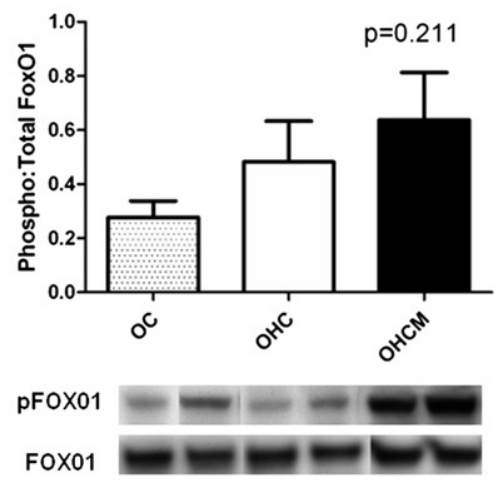

FIGURE 2. Protein expression in ischemic myocardium. The ratio of phosphorylated to total protein expression calculated for proteins in the insulin signaling pathway. *Bonferroni $P<.001$. IRS1, Insulin receptor substrate 1; IRS2, insulin receptor substrate 2; pIRS1, phosphorylated insulin receptor substrate 1; pIRS2, phosphorylated insulin receptor substrate $2 ; A K T$, protein kinase $\mathrm{B} ; p A K T$, phosphorylated protein kinase $\mathrm{B} ;$ MTOR, mammalian target of rapamycin; $p M T O R$, phosphorylated mammalian target of rapamycin; $A M P K$, adenosine monophosphate-activated protein kinase; $p A M P K$, phosphorylated adenosine monophosphate-activated protein kinase; FOXO1, foxhead box 01; pFOX01, phosphorylated foxhead box 01; OC, Ossabaw control; OHC, Ossabaw high cholesterol; OHCM, Ossabaw high cholesterol with metformin.

the OHCM group. Although tyrosine phosphorylation activates IRS, serine phosphorylation by insulin receptor kinase inhibits IRS, thereby inhibiting insulin signal transduction. Analysis of phosphorylated-to-total IRS1 and -IRS2 ratios were not significant among groups, and despite upregulation of p-IRS2 in the OHCM group, insulin signaling was not inhibited, as evidenced by the upregulation of downstream targets including PI3K and AKT. Phosphoinostitide 3-kinase expression was upregulated in both the $\mathrm{OHC}$ and OHCM groups compared with the OC group; however, only the OHCM group had increased expression of its downstream target: AKT. The phosphorylated AKT (Ser 473)-to-AKT ratio was also markedly elevated in the OHCM group, suggesting that metformin either increases phosphorylated AKT phosphorylation or decreases the activity of phosphatases. Similarly, the dramatic upregulation of p-MTOR in the OHCM group suggests that metformin augments the intrinsic catalytic activity in MTOR, resulting in increased autophosphorylation at Ser 2481 and enhanced insulin signaling.
Interestingly, although there was a substantial upregulation of AMPK, there was also upregulation of MTOR and p-MTOR despite the fact that AMPK is a known inhibitor of MTOR. Ribosomal protien S6 is a downstream target of MTOR. When MTOR is activated, it leads to phosphorylation of S6 and is considered a marker for MTOR activity. This study demonstrated that although S6 expression was unchanged, there was a significant downregulation of pS6 expression in the OHCM group. Deptor is another potent inhibitor of MTOR activity, and there was an increase in Deptor expression in the OHCM group. Taken together, these results suggest that although MTOR and p-MTOR expression are increased in the OHCM group, MTOR activity is decreased likely because of Deptor- and AMPK-mediated MTOR inhibition.

AKT also phosphorylates and inactivates FOX01, a member of the forkhead box transcription factor family, which promotes gluconeogenesis in a fasted state. In a well-fed state, when insulin levels are high, phosphorylated FOX01 is excluded from the nucleus, thereby reducing hepatic 

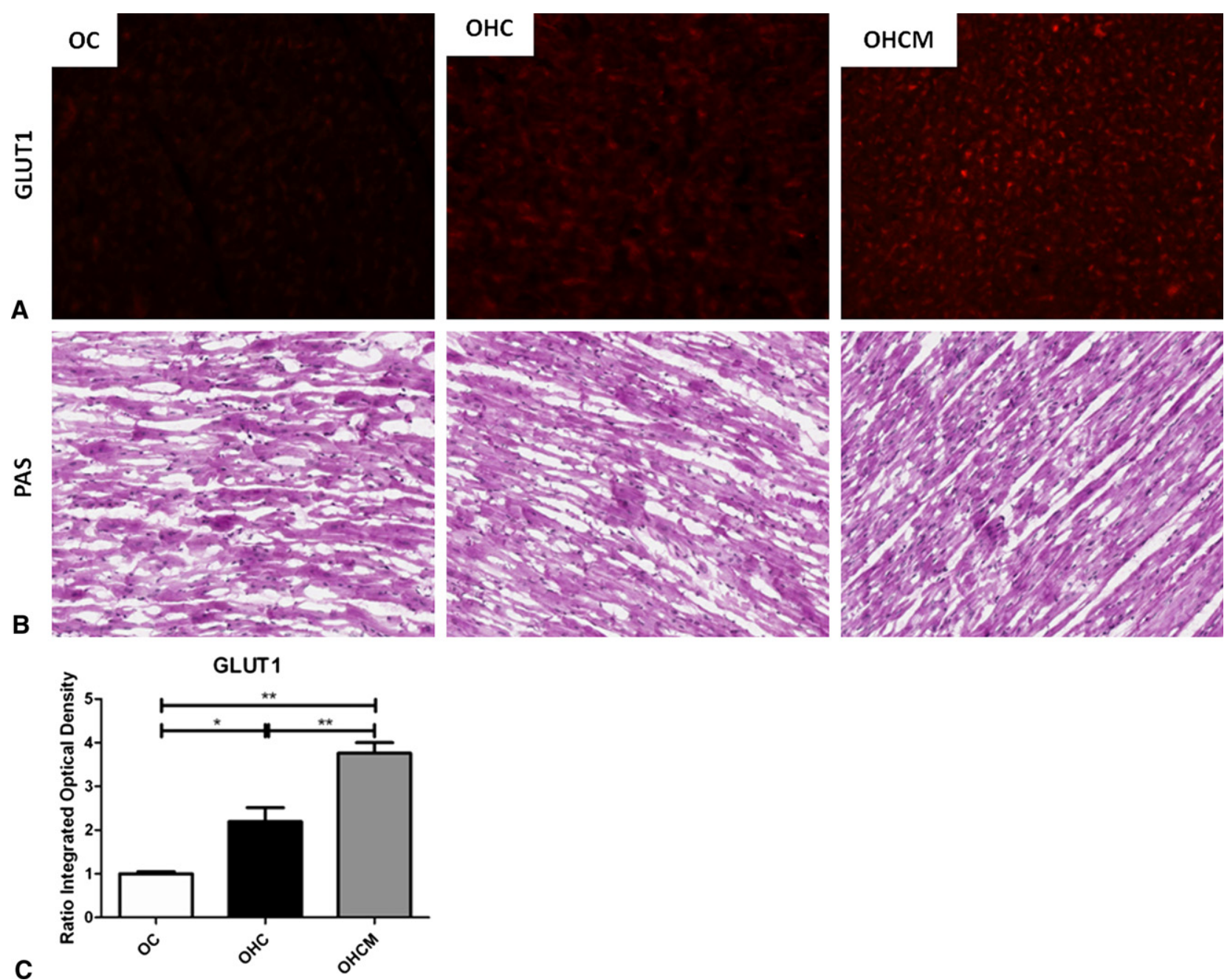

FIGURE 3. Histologic analysis of ischemic cardiac tissue. A, Immunofluorescence staining for GLUT1. Red staining represents GLUT1. B, Periodic acidSchiff staining for cardiomyocyte glycogen stores. C, GLUT1 density analysis. *Bonferroni $P<.01$. **Bonferroni $P<.001$. OC, Ossabaw control; $O H C$, Ossabaw high cholesterol; OHCM, Ossabaw high cholesterol with metformin; GLUT1, glucose transporter 1; PAS, periodic acid-Schiff.

glucose production. ${ }^{15}$ Both the $\mathrm{OHC}$ and $\mathrm{OHCM}$ groups had elevated FOX01 and phosphorylated FOX01 levels, suggesting the hypercholesterolemic diet and elevated baseline insulin levels signaled the phosphorylation and inactivation of FOX01.

One of the key mechanisms of action of metformin is mediated by AMPK activation altering the adenosine monophosphate (AMP)-to-adenosine triphosphate (ATP) ratio in the liver, which effectively inhibits hepatic glucose production. ${ }^{16}$ Metformin-mediated increases in AMP binds AMPK and exposes the catalytic domain to the AMPK kinase, which facilitates AMPK phosphorylation and subsequent activation. ${ }^{17}$ In this study, the OHCM group had a 30-fold increase in phosphorylated AMPK expression, which is consistent with the known mechanism of action of metformin. Despite the marked upregulation of AMPK, this did not result in the expected metabolic shift from malonyl CoA to acetyl CoA and the resultant shift to fatty acid metabolism. There was no difference in ACC, phosphorylated ACC, or FAS expression; therefore, it does not appear that the increased expression of AMPK impacted fatty acid synthesis.

Transmembrane glucose transport is mediated by glucose transporter family GLUT. In the heart, GLUT1 and GLUT4 are the primary glucose transporters, with GLUT1 responsible for basal glucose transport and GLUT4 responsible for insulin-activated glucose transport. GLUT4 is stored in transport vesicles and translocates to the plasma membrane in an inducible fashion on insulin stimulation or with increased cardiac contractility. ${ }^{18}$ GLUT4 translocation to the plasma membrane can increase glucose uptake swiftly into the cell. Of note, GLUT1 is also stored in transport vesicles, and translocation to the plasma membrane is also dependent on insulin signaling and contraction. ${ }^{19}$ Animal studies have shown that under conditions of hypoxia and altered AMP-to-ATP ratio, AMPK 


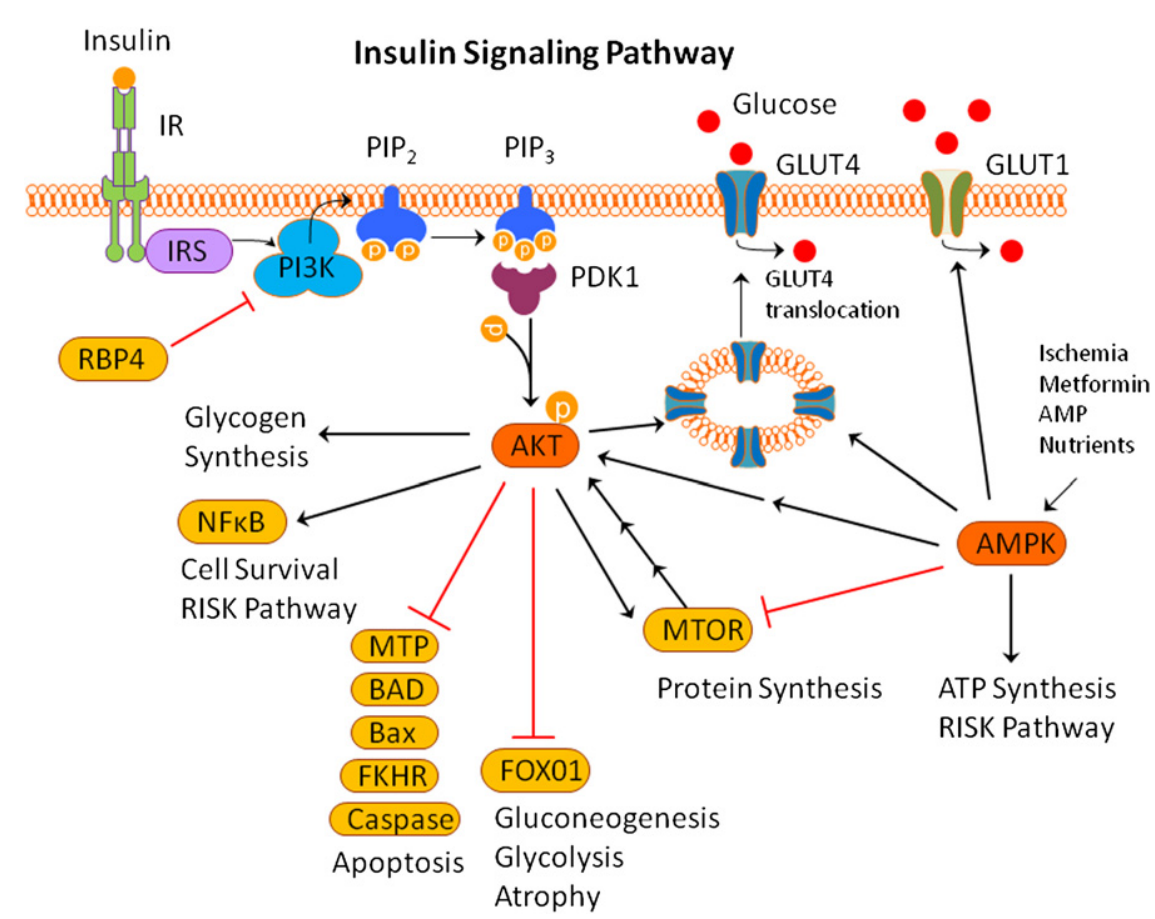

FIGURE 4. Insulin signaling transduction. The effects of insulin on glucose uptake and apoptosis/survival presented here are discussed in detail in the text. $M T P$, Mitochondrial transition pore; FKHR, forkhead transcription factors; IRS, insulin receptor substrate; AKT, protein kinase B; MTOR, mammalian target of rapamycin; $A M P K$, adenosine monophosphate-activated protein kinase; $R B P 4$, retinol binding protein 4; $A M P$, adenosine monophosphate; $I R$, insulin receptor; p13k, phosphoinostitide 3-kinase; PIP2, phosphatidylinostitol biphosphate; PIP3, phosphatidylinostitol triphospate; PDK1, 3-phosphoinostitide-dependent kinase 1; GLUT4, glucose transporter 4; GLUT1, glucose transporter 1; NFkB, nuclear factor kappa B; BAD, Bcl-2-associated death promoter; BAX, Bcl-2-associated X protien; FOX01, foxhead box 01.

is activated, resulting in increased GLUT1-mediated glucose transport. Adenosine monophosphate-activated protein kinase activation unmasks preexisting GLUT1 transporters on the plasma membrane, which substantially enhances glucose transport. ${ }^{20}$ The increase in glucose transport does not correlate with changes in the plasma membrane levels of GLUT1. ${ }^{21}$

Interestingly, although metformin upregulated insulin signaling, in this study there was no change in GLUT4 expression in the $\mathrm{OHC}$ and $\mathrm{OHCM}$ groups compared with the OC group. However, the OHCM group demonstrated dramatic upregulation of GLUT1 expression at the plasma membrane compared with the OHC and OC groups, resulting, perhaps, from the combination of insulin and AMPK-mediated GLUT1 translocation to the plasma membrane. Alternatively, the increased GLUT1 expression at the plasma membrane may be the result of decreased endocytosis of GLUT1 vesicles back to the cytosol. Previous studies have examined the role of GLUT1-mediated glucose uptake in the prevention of apoptosis and have shown that overexpression of GLUT1 prevented hypoxia-induced apoptosis independent of extracellular glucose concentration. ${ }^{22}$ Given the findings in this study, GLUT1 warrants further investigation because it may be yet another mechanism by which metformin is cardioprotective.
Retinol binding protein 4 is a protein secreted by adipocytes that inhibits PI3K-mediated insulin signaling and increases hepatic glucose production. Animal and human studies have shown that elevated serum RBP4 levels correlate closely with type 2 diabetes mellitus and other insulin-resistant states. ${ }^{23}$ In this study, myocardial RBP4 expression was increased in both the $\mathrm{OHC}$ and $\mathrm{OHCM}$ groups; however, only the OHCM group was significant. Interestingly, the OHCM group demonstrated the highest expression of RBP4 and its target PI3K, suggesting that, perhaps, the metformin-mediated insulin signaling propagation outweighs the inhibitory effect of RBP4 on PI3K. Another possibility is that RBP4 is not as effective in the myocardium in inhibiting PI $3 \mathrm{~K}$ as it is in its primary tissue targets-namely, skeletal muscle, adipose, and liver.

Although cardioprotective mechanism of metformin is not understood completely, it appears that AMPK and AKT are important in mediating these effects. Previous studies have shown that metformin-induced PI3K-AKT upregulation inhibits mitochondrial permeability transition pore during periods of ischemia, thereby limiting reperfusion injury. ${ }^{24}$ Phosphoinostitide 3-kinase and AKT are integral protein kinases in the reperfusion injury salvage kinase family that promote cell survival and cardioprotection after an ischemic insult. Adenosine monophosphate-activated 
protein kinase also activates the reperfusion injury salvage kinase pathway and initiates ischemic preconditioning. ${ }^{10}$ Metformin-mediated AMPK activation has also been shown to increase ATP generation and attenuates cardiomyocyte apoptosis. ${ }^{17}$ Thus, metformin effectively preconditions the heart against ischemia-reperfusion injury by activating AMPK. Our findings demonstrate that chronic treatment with metformin in the context of metabolic syndrome and myocardial ischemia dramatically upregulate insulin signaling, which is at the crossroads of known metabolic and survival benefits of metformin.

\section{References}

1. Centers for Disease Control and Prevention. National diabetes fact sheet: national estimates and general information on diabetes and prediabetes in the United States. Atlanta, GA: U.S. Department of Health and Human Services, Centers for Disease Control and Prevention, 2011; 2011.

2. Grundy MS, Cleeman JI, Daniels SR, Donato KA, Eckel RH, Franklin BA, et al. Diagnosis and management of the metabolic syndrome: an American Heart Association/National Heart, Lung, and Blood Institute Scientific Statement. Circulation. 2005;112:2735-52.

3. Mente A, Yusuf S, Islam S, McQueen M, Tonomsup S, Onen C, et al. Metabolic syndrome and risk of acute myocardial infarction: a case-control study of 26,903 subjects from 52 countries. J Am Coll Cardiol. 2010;55:2390-8.

4. Isomaa B, Almgren P, Tuomi T, Isomaa B, Lahti K, Nissen M, et al. Cardiovascular morbidity and mortality associated with the metabolic syndrome. Diabetes Care. 2001;24:683-9.

5. Wilson PW, Kannel WB, Silbershatz H, D'Agostino RB. Clustering of metabolic factors and coronary heart disease. Arch Intern Med. 1999;159:1104-9.

6. Kim J, Montagnani M, Koh KK, Quon MJ. Reciprocal relationships between insulin resistance and endothelial dysfunction: molecular and pathophysiological mechanisms. Circulation. 2006;113:1888-904.

7. Molavi B, Rassouli N, Bagwe S, Rasouli N. A review of thiazolidinediones and metformin in the treatment of type 2 diabetes with focus on cardiovascular complications. Vasc Health Risk Manage. 2007;3:967-73.

8. Johnson JA, Majumdar SR, Simpson SH, Toth EL. Decreased mortality associated with the use of metformin compared with sulfonylurea monotherapy in type 2 diabetes. Diabetes Care. 2002;25:2244-8.

9. UK Prospective Diabetes Study Group. Effect of intensive blood-glucose control with metformin in complications in over-weight patients with type 2 diabetes (UK-PDS 34). Lancet. 1998;352:837-53.

10. ElMessaoudi S, Rongen GA, de Boer RA, Riksen NP. The cardioprotective effects of metformin. Curr Opin Lipidol. 2011;22:445-53.

11. Lassaletta AD, Chu LM, Robich MP, Elmadhun NY, Feng J, Burgess TA, et al. Overfed Ossabaw swine with early stage metabolic syndrome have normal coronary collateral development in response to chronic ischemia. Basic Res Cardiol. 2012;107:1-11.

12. National Research Council (U.S.), Committee for the Update of the Guide for the Care and Use of Laboratory Animals, Institute for Laboratory Animal Research (U.S.), National Academies Press (U.S.). Guide for the care and use of laboratory animals. Washington, DC: National Academies Press; 2011.

13. Bertrand L, Horman S, Beauloye C, Vanoverschelde JL. Insulin signaling in the heart. Cardiovasc Res. 2008;79:238-48.

14. Sykiotis GP, Papavassiliou AG. Serine phosphorylation of insulin receptor substrate-1: a novel target for the reversal of insulin resistance. Mol Endocrinol. 2001;15:1864-9.

15. Zhang W, Patil S, Chauhan B, Guo S, Powell DR, Le J, et al. Fox01 regulates multiple metabolic pathways in the liver. J Biol Chem. 2006;281:10105-17.

16. Zhou G, Myers R, Li Y, Chen Y, Shen X, Fenyk-Melody J, et al. Role of AMP-activated protein kinase in mechanism of metformin action. J Clin Invest. 2001;108:1167-74.

17. Calvert JW, Sundewar S, Jha S, Greer JM, Bestermann WH, Tian R, et al. Acute metformin therapy confers cardioprotection against myocardial infarction via AMPK-eNOS-mediated Signaling. Diabetes. 2008;57:696-705.

18. Schwenk RW, Luiken JJ, Bonen A, Glatz JF. Regulation of sarcolemmal glucose and fatty acid transporters in cardiac disease. Cardiovasc Res. 2008;79: 249-58.
19. Wheeler TJ, Fell RD, Hauck MA. Translocation of two glucose transporters in heart: effects of rotenone, uncouplers, workload, palmitate, insulin and anoxia. Biochem Biophys Acta. 1994;1196:191-200.

20. Abbud W, Habinowski S, Zhang J, Kendrew J, Elkairi FS, Kemp BE. Stimulation of AMP-activated protein kinase is associated with enhancement of Glut1mediated glucose transport. Arch Biochem Biophys. 2000;380:347-52.

21. Barnes K, Ingram JC, Porras OH, Barros LF, Hudson ER, Fryer LG, et al. Activation of GLUT1 by metabolic and osmotic stress: potential involvement of AMP-activated protein kinase. J Cell Sci. 2002;115:2433-42.

22. Moley KH, Mueckler MM. Glucose transport and apoptosis. Apoptosis. 2000;5: 99-105.

23. Graham TE, Yang Q, Bluher M, Hammarstedt A, Ciaraldi T, Henry RR, et al. Retinol binding protein 4 and insulin resistance in lean, obese, and diabetic subjects. N Engl J Med. 2006;354:2552-63.

24. Bhamra GS, Hausenloy DJ, Davidson SM, Carr RD, Paiva M, Wynne AM, et al Metformin protects the ischemic heart by the Akt-mediated inhibition of mitochondrial permeability transition pore opening. Basic Res Cardiol. 2008;103 274-84.

\section{Discussion}

Dr Harold L. Lazar (Boston, Mass). I enjoyed your presentation and thank you for allowing me to see the manuscript in advance. I just have a couple of small questions to ask: How did you choose the dose of metformin? Five hundred milligrams fed orally twice a day is considered a smaller dose. Do you think there is any dose-response relationship with what you saw?

Dr Elmadhun. We chose that dose based on human dosing. And actually, these animals are smaller-they're about $30 \mathrm{~kg}$ and so we anticipate that these levels would be synonymous with what we find in humans.

Dr Lazar. I'm also interested-. Did you think about giving the metformin prior to the ischemia for a period of time? I' $m$ just wondering if it would have been interesting to see what the signaling pathways, the expression of GLUT4, might have been increased if you had given it prior to performing the period of ischemia.

Dr Elmadhun. Certainly, that would be interesting to look at in future studies-to administer metformin prior to chronic ischemia or in an acute ischemia-reperfusion model. In other studies, that would be interesting to do, too. As opposed to a chronic model of ischemia, as the prior speakers discussed, an acute reperfusion injury would also be an interesting thing to look at as well.

Dr Lazar. Have you also considered looking at the effects of metformin in other cardioprotective agents such as endothelial nitric oxide synthase, which is also mediated through many of these pathways?

Dr Elmadhun. Yes. Actually, I just presented that data at the Atherosclerosis, Thrombosis, Vascular Biology last week, and we did find that there were other cardioprotective mechanisms by increasing cell protective proteins and also reduction or inhibition of apoptosis-related proteins. We did find that there was upregulation of phosphorylated endothelial nitric oxide synthase in the ischemic territories.

Dr Lazar. And finally, did you look at any changes in either hemodynamic function or cardiac function contractility in this model?

Dr Elmadhun. We did. We looked at contractility and hemodynamic parameters, and we did not find any differences among groups.

Dr Thomas K. Waddell (Toronto, Ontario, Canada). I would like to ask you a question about study design. I am sure it is 
expensive to do these kinds of studies, but I was really struck by the figure where you showed activation of the AMPK in the animals that received metformin where there was actually relatively little difference between the normal animals and the metabolic syndrome animals. What would you think, or maybe other people have already examined it, would be the effect of metformin on animals without metabolic syndrome?
Dr Elmadhun. Previous studies have looked at this-looking at just animals with a regular diet and supplemented with metformin to prove the fact that patients don't need to have diabetes mellitus with metabolic syndrome and tolerate metformin — and they have found that one of the mechanisms is to trick cells into believing that they are starved and increasing the AMP-to-ATP ratio, which thereby activates AMPK. 